\title{
Chronic periodontitis and herpes viruses
}

\begin{abstract}
Chronic periodontitis is the most common form of periodontitis, and is prevalent in adults, but can be observed in children. It is associated with the accumulation of plaque and calculus and has a slow to moderate rate of disease progression, but periods of more rapid destruction may be observed. Different factors have been involved in the initiation of periodontitis in an individual including gene polymorphism, bacterial, immunological and environmental causes. Recently however, different viruses were detected in chronic periodontitis patients. In this mini review we discuss the role of the herpes viruses in the development of chronic periodontitis.
\end{abstract}

\author{
Volume I Issue 2 - 2014
}

\author{
Mahmoud YM Taha,' Zeyad T Al Rassam² \\ 'Department of Dental Science, Mosul University, Iraq \\ ${ }^{2}$ Department of Biology, Mosul University, Iraq
}

Correspondence: Mahmoud YM Taha, College of Dentistry,

Mosul University, Iraq, Email tahadent04@yahoo.com

Received: May 13, 2014 | Published: June 05, 2014

Keywords: Chronic periodontitis, Herpes viruses

\section{Introduction}

\section{Periodontitis}

Periodontitis is a group of illnesses located in the gingiva and other dental supporting tissues. ${ }^{1}$ Traditionally, periodontitis has been described as a bacterial infection caused by anaerobic bacteria including Porphyromonas ginivalis, Tannerella forsythia, prevotella intermedia, Aggregatibacter actinomycetemcomitans, Fusibacterium nucleatum and campylobacter rectus ${ }^{2}$ that initiate microbial plaque, which accumulates on the tooth surface at the gingival margin and in periodontal pockets. ${ }^{3}$ Moreover, chronic inflammation in gingiva and connective tissues may eventually result in tooth loss. ${ }^{1}$ Periodontitis is most often seen in maturity, with the majority of adults experiencing some signs and symptoms. ${ }^{4}$

Individuals with periodontal lesions may harbor millions of genomic copies of herpes viruses, papilloma viruses, human immunodeficiency virus (HIV), human T-lymph tropic virus type 1, torqueteno virus, and hepatitis $\mathrm{B}$ and $\mathrm{C}$ viruses, ${ }^{5}$ as they were detected in periodontal infections. ${ }^{6}$ Thus, reactivation of these viruses may initiate or accelerate periodontal tissue destruction by lytic activity against periodontal cells, immune mediated tissue destruction and immune suppression, which elevates the susceptibility of the host to bacterial attacks and increases virulence of local pathogenic bacteria. ${ }^{7-9}$ Therefore, the evolution of periodontal disease depends upon: periodontopathic properties such as virulence factors and anaerobiosis; local host immune responses that activate innate immune system cells which include macrophages, dendritic cells, natural killer cells, neutrophils, osteoclasts and furthermore humeral response via B-cells: oral cavity environmental changes such as smoking, diabetes and nutrition. ${ }^{10-13}$

\section{Herpes viruses}

The term herpes is derived from the Greek word meaning "to creep," reflecting clinical observations of latent recurring infections that progress slowly. ${ }^{14}$ Herpes viruses are large double-stranded DNA viruses widely dispersed in nature and associated with many human diseases. ${ }^{15}$ The human herpes viruses share four significant biological properties:

a. They encode specific enzymes involved in the biosynthesis of viral nucleic acids. These enzymes are genetically distinct from the host enzymes and provide unique therapeutic targets for inhibition by antiviral agents. ${ }^{16,17}$

b. The synthesis of viral DNA is initiated in the nucleus, and assembly of the capsid is also initiated in the nucleus. c. Release of progeny virus from the infected cell is accompanied by cell death.

d. They establish latent infection within tissues that are distinct for each virus, and latency is established lifelong in the host. ${ }^{18}$

\section{Herpes viruses and periodontal disease}

Herpes viruses are very complex; more than $90 \%$ of the world's population is infected with human herpes viruses. Most infections with these viruses usually occur in childhood via infected secretions such as saliva. Primary infection with herpes viruses may cause oral mucosal lesions, and / or entry into indefinite latency with the viruses reactivated under various conditions. The main cause of reactivation is immune suppression and these infections may cause severe diseases in HIV infection and other immuno compromised patients. ${ }^{19}$ Since the mid-1990s, herpes viruses have emerged as putative pathogens in chronic and aggressive periodontitis as well as gingivitis. ${ }^{20}$ Herpes viruses are often found in periodontal pockets and may initiate or accelerate periodontal tissue destruction by lytic activity against periodontal cells, immune mediated tissue destruction and immune suppression, which elevates the susceptibility of the host to bacterial attacks and increases virulence of local pathogenic bacteria. ${ }^{9}$

Herpes virus infected periodontal sites tend to show tissue breakdown more frequently than herpes virus free sites, and this active infection is associated with increased risk of progressive periodontal disease. ${ }^{6,7}$

\section{Herpes virus behavior in periodontium}

Several studies have suggested an increased frequency of detection of specific members of the herpes viridae family, such as EBV, HCMV and HSV in various forms of periodontal disease including gingivitis, localized or generalized chronic and aggressive periodontitis., ${ }^{2,6,21}$ Herpes viruses may exert periodontopathic potential through several mechanisms:

a. In periodontitis lesions herpes viruses may have a direct effect by lytic activity on the fibroblasts, keratinocytes, endothelial cells, lymphocytes, bone cells and inflammatory cells in the periodontium. $^{22}$

b. Herpes viruses can reduce the host defense system via infecting and altering the functions of monocytes, macrophages, and lymphocytes as antigen presenting cells in periodontitis lesions and this effect may hamper tissue turnover and repair. ${ }^{23}$ Herpes virus infection may increase the pathogenicity of the periodontal microorganism by enhancing bacterial adherence 
to periodontal pocket epithelial cells via creating new bacterial binding sites.

c. Periodontal herpes viral infections can increase and alter inflammatory mediator and cytokine responses, which can up regulate interleukin 1-beta (IL-1 $\beta$ ) and tumor necrosis factor-alpha (TNF- $\alpha$ ) gene expression in monocytes and macrophages. ${ }^{6,24,25}$

d. Herpes viruses can induce tissue damage via immuno pathological responses to virally infected cells. Herpes viruses can induce cell mediated immuno suppression by reducing the cell surface expression of MHC I molecules, thereby interfering with T-lymphocyte recognition. ${ }^{24}$

e. Periodontal herpes virus infected sites seem to be associated with increased levels of periodontal bacterial pathogens especially anaerobic bacteria and tend to exhibit more damage to periodontal tissue than herpes virus free sites, therefore, herpes viral active infections are related with an elevated risk of progressive periodontal disease..$^{22}$

\section{Conclusion}

Different studies have detected many viruses including herpes viruses in periodontal pockets suggesting a role for the existing viruses in the progression of periodontal disease. This should be further clarified by in situ hybridization and immune peroxidase staining to identify specific viral antigens in periodontal cells.

\section{Acknowledgment}

None.

\section{Conflict of interest}

None.

\section{References}

1. Scheres N, Laine ML, de Vries TJ, et al. Gingival and periodontal ligament fibroblasts differ in their inflammatory response to viable Porphyromonas gingivalis. J Periodontal Res. 2010;45(2):262-270.

2. Saygun I, Kubar A, Sahin S, et al. Quantitative Analysis of Association between herpes viruses and bacteria in periodontitis. $J$ Periodontal Res. 2008;43(3):352-359.

3. Jansson H. Studies on periodontitis and analyses of individuals at risk for periodontal diseases. Swed Dent J Suppl. 2006;180:5-49.

4. Bascones MA, Figuero RE. Periodontal diseases as bacterial Infection. Av Periodon Implantol. 2005;17(3):111-118.

5. Slots J. Oral viral infections of adults. Periodontol 2000. 2009;49:60-86.

6. Slots J. Herpesviruses in periodontal diseases. Periodontol 2000. 2005;38:33-62.

7. Lin YL, Li M. Human cytomegalovirus and Epstein-Barr virus inhibit oral bacteria-induced macrophage activation and phagocytosis. Oral Microbiol Immunol. 2009;24(3):243-248.
8. Botero JE, Parra B, Jaramillo A, et al. Subgingival Human Cytomegalovirus (HCMV) correlates with increased clinical periodontal parameters and bacterial co-infection in periodontitis. $J$ Periodontol. 2007;78(12):2303-2310.

9. Slots J. Herpesviral-bacterial synergy in the pathogenesis of human periodontitis. Curr Opin Infect Dis. 2007;20(3):278-283.

10. Abiko Y, Sato T, Mayanagi G, et al. Profiling of subgingival, plaque biofilm microflora from periodontally healthy subjects and from subjects with periodontitis using quantitative real-time PCR. $J$ Periodontal Res. 2010;45(3):389-395.

11. Chuang HC, Lay JD, Hsieh WC, et al. Pathogenesis and mechanism of disease progression from hemophagocytic lymphohistiocytosis to EpsteinBarr virus-associated T-cell lymphoma: nuclear factor-kappaB pathway as a potential therapeutic target. Cancer Sci. 2007;98(9):1281-1287.

12. Watanabe SA, Correia-Silva Jde F, et al. EBV-1 and HCMV in aggressive periodontitis in Brazilian patients. Braz Oral Res. 2007;21(4):336-341.

13. Takahashi N. Microbial ecosystem in the oral cavity: metabolic diversity in an ecological niche and its relationship with oral diseases. Int Cong Ser. 2005; 1284:103-112.

14. Willey JM, Sherwood LM, Woolverton CJ. Prescott's principles of microbiology. (1st Edn), McGraw-Hill Companies, Avenue of the Americas, New York. 2009;pp.565-566,684-686.

15. McGeoch DJ, Dolan A, Ralph AC. Toward a comprehensive phylogeny for mammalian and avian herpes viruses. J Virol. 2000;74(22):10401-10406.

16. Carter J, Saunders V. Virology Principles and Applications. John Wiley \& Sons Ltd., Chichester, England. 2007;pp.122-135.

17. Roizman B, Pellett $P$. The Family of Herpes viridae: A Brief Introduction. Knipe DM, Howley PM (Eds.), Fields-Virology, (4th edn), Chapter 71, Lippincott Williams \& Wilkins Publishers. 2001;pp.1929-1939.

18. Kudesia G, Wreghitt T. Clinical and Diagnostic Virology. (1st edn), Cambridge University Press, UK. 2009.

19. Robinson PG, Sheiham A, Challacombe SJ, et al. The periodontal health of homosexual men with HIV infection: a controlled study. Oral Dis. 1996;2(1):45-52.

20. Contreras A, Slots J. Herpesviruses in human periodontal disease. $J$ Periodontal Res. 2000;35(1):3-16.

21. Kolliyavar B, Setty S, Patil A, et al. Association of viruses in chronic periodontitis - fact or hype??? Int J Oral Hyg \& Dent Res. 2013;1(1):1-5.

22. Pushpa SP, Soumya BG. Herpesviruses in Human Periodontal disease. $J$ Int Oral Health. 2010;2(2):59-63.

23. Contreras A, Zadeh H, Nowzari H, et al. Herpes virus infection of inflammatory cells in human periodontitis. Oral Microbiol Immunol. 1999;14(4):206-212.

24. Slots J. Human viruses in periodontitis. Periodontal 2000. 2010;53:89-110.

25. Ledder RG, Gilbert P, Huws SA, et al. Molecular analysis of the subgingival microbiota in health and disease. Appl Environ Microbiol. 2007;73(2):516-523. 